because without a variable threshold, the standard for capacity would be the same for all decisions (and not decision-specific).

Finally, although the US government has not ratified the United Nations Convention on the Rights of Persons with Disabilities, American physicians certainly agree that their ethical duty when assessing capacity is to assess the patient's abilities and, where possible, assist incapacitated patients in regaining capacity. The American psychiatric literature is replete with exhortations to restore capacity or enhance decision-making abilities following a finding of incapacity. ${ }^{7}$ We hope that our editorial provides guidance on one aspect of that process of assessment and assistance.

1 Berg JW, Appelbaum PS, Grisso T. Constructing competence: formulating standards of legal competence to make medical decisions. Rutgers Law Rev 1996; 48: 345-71.

2 Appelbaum PS. Assessment of patients' competence to consent to treatment. N Engl J Med 2007; 357: 1834-40

3 Law Reform Commission. Report No 231: Report on Mental Incapacity. HMSO, 1995.

4 Oxford English Dictionary. Grasp. Oxford University Press, 2019 (http://www. oed.com/view/Entry/80878?rskey=S8Bacb\&result=2\&isAdvanced=false).

5 Appelbaum PS, Grisso T. Assessing patients' capacities to consent to treatment. N Engl J Med 1988; 319: 1635-8.

6 Ryan C, Callaghan S, Peisah C. The capacity to refuse psychiatric treatment: a guide to the law for clinicians and tribunal members. Aust $N Z J$ P Psychiatry 2015; 49: 324-33.

7 Kim SYH. Evaluation of Capacity to Consent to Treatment and Research. Oxford University Press, 2010.

Rocksheng Zhong, Lecturer, Department of Psychiatry, Yale School of Medicine, USA Dominic A. Sisti, Assistant Professor, Department of Medical Ethics and Health Policy, University of Pennsylvania; and Director of the Scattergood Program for Applied Ethics of Behavioral Health Care, Perelman School of Medicine, University of Pennsylvania, USA: Jason H. Karlawish, Professor, Departments of Medicine, Medical Ethics and Health Jason H. Karlawish, Professor, Departments of Medicine, Medical Ethics and Health
Policy, and Neurology, University of Pennsylvania; and Co-Director of the Penn Memory Center, Perelman School of Medicine, University of Pennsylvania, USA.

Email: rocksheng.zhong@yale.edu

doi:10.1192/bjp.2019.143

\section{CORE study: different interpretation of the results}

Lloyd-Evans et al ${ }^{1}$ published results from a cluster-randomised trial looking at the effect on patients of an improvement programme for mental health crisis resolution teams, in which the aim was to increase fidelity with the crisis resolution team model. In the intervention group, the authors found a reduction in admissions and in-patient bed days but no increase in average patient satisfaction. We have two comments about interpretation of their results.

First, the authors report that there was no difference in average patient satisfaction score between the intervention and the control group. They offer a ceiling effect as a possible explanation, given that average patient satisfaction was already high before the intervention. We wonder whether this ceiling effect can be at least partially explained by the timing of their assessment? The authors measured patient satisfaction around the time of discharge from the home treatment team. Patient satisfaction, however, tends to be lower if the time interval between intervention and measurement is larger. ${ }^{2}$ The Mind report, Listening to Experience ${ }^{3}$ - cited by the authors - suggests that patients are far more critical about crisis care, when questioned at a much later date following discharge. Studies reporting patient satisfaction 6 months or longer after the crisis episode are desperately needed.

Second, there remains the question of whether the observed reduction in admissions and in-patient bed days found in the intervention group is related to an increase in the fidelity scores. The crisis resolution teams in the intervention group received additional support to increase both their fidelity to the model and their scores on the fidelity scale. And yet despite this, the authors also mention in the article, and in the supplementary material (pp. 47-50), that there is no relationship between the fidelity scale scores and the reduction in admissions and in-patient bed days.

This makes us wonder about what are the causal factors in reducing admissions and in-patient bed days? It seems that an increase in scores on the fidelity scale is not necessarily essential to achieving this. This observation is important for us as practicing clinicians. The results here suggest that we ought to be aiming to secure the actual intervention itself, namely the access to a facilitator, the opportunity to discuss team improvement at a specially arranged day and the development of a service improvement plan and not be focusing on getting higher scores on the fidelity scale.

1 Lloyd-Evans B, Osborn D, Marston L, Lamb D, Ambler G, Hunter R, et al. The CORE service improvement programme for mental health crisis resolution teams: results from a cluster-randomised trial. Br J Psychiatry 2019; xx:xx-xx.

2 Jensen HI, Ammentorp J, Kofoed PE. User satisfaction is influenced by the interval between a health care service and the assessment of the service. Soc Sci Med 2010; 70: 1882-7.

3 Mind. Listening to Experience: An Independent Inquiry into Acute and Crisis Mental Health Care. Mind, 2011.

Pang Loong Wong, Specialty Registrar in Psychiatry, South West London and S George's NHS Trust, UK; Robert Bertram, Psychiatrist, South West London and St George's NHS Trust, UK; Dieneke Hubbeling, Psychiatrist, South West London and St George's NHS Trust, UK. Email: Adrian.Wong@swlstg.nhs.uk

doi:10.1192/bjp.2019.144

\section{Authors' reply}

We agree with the thoughtful letter by Wong and colleagues up to a point. The Crisis team Optimisation and RElapse prevention (CORE) Fidelity Scale for crisis resolution teams (CRTs) was based mainly on stakeholders' opinions rather than robust empirical evidence regarding components of effective crisis care. ${ }^{1}$ Some fidelity items may be more important than others, and some items may not constitute critical ingredients of effective CRTs.

The CORE service improvement programme evaluated in our trial $^{2}$ built in a lot of flexibility and ownership for teams to choose their own goals for improving their service and plan how these would be achieved, in their local context, given their available resources. This flexibility in the programme was valued by the teams. We agree that giving CRT teams dedicated time and space to reflect on their team's performance and how this could be improved, and offering support from an experienced clinician (the CRT facilitator), are both important components of the programme.

We do not recommend that practitioners should ignore CRT model fidelity, however, for two reasons. First, the CORE CRT Fidelity Scale specifies many aspects of CRT service organisation and delivery, and the total fidelity score is a fairly blunt measure. Although our trial found no relationship between CRT total fidelity score and hospital admission or CRT patients' readmission rates, we did find relationships between these outcomes and fidelity scale subscale scores, as reported in our paper. ${ }^{2}$ Our results suggest that to avert hospital admissions requires rapid, easy access to CRT care (the access and referrals subscale); while to help CRT patients recover and avoid readmissions to acute care requires provision of good quality CRT care (the content of care, and timing and location of care subscales). This makes intuitive and clinical sense. Different fidelity items may be most important for different outcomes but are diluted in the total fidelity score.

Second, seeking to improve model fidelity was an integral part of our trial's successful CRT service improvement programme. CRT teams' whole-team scoping day and their service improvement 
plans were informed by a fidelity review. Teams targeted specific team) as the means by which to improve their service. Our trial by a CRT fidelity review and focused on improving model fidelity, was successful in reducing hospital admissions and CRT patients' readmissions to acute care. Wong and colleagues' suggestion that this could be achieved just as successfully without reference to model fidelity is an untested assertion.

Our exploration of the relationship between CRT Fidelity Scale scores and outcomes involved only 25 teams in the unusual context of a trial. Further research is desirable to establish the relationship between model fidelity and outcomes, and, in time ideally, to refine the CRT Fidelity Scale to include only items demonstrated to constitute critical components of the CRT model.

In the meantime, the CORE CRT Fidelity Scale may not provide a blueprint, but does offer a helpful guide for practitioners and service planners in what an effective, high-quality CRT service looks like. As such, it is recognised as a descriptor of best practice for CRTs in current NHS England policy guidance. ${ }^{3}$

1 Lloyd-Evans B, Bond G, Ruud T, Ivanecka A, Gray R, Osborn D, et al. Development of a measure of model fidelity for mental health crisis resolution teams. BMC Psychiatry 2016; 16: 427.

2 Lloyd-Evans B, Osborn D, Marston L, Lamb D, Ambler G, Hunter R, et al. The CORE service improvement programme for mental health crisis resolution teams: results from a cluster-randomised trial. Br J Psychiatry 2019; doi: https://doi.org/10.1192/bjp.2019.21

3 NHS England. Crisis and Acute Care for Adults. NHS England, no date (https:// www.england.nhs.uk/mental-health/adults/crisis-and-acute-care/)

Brynmor Lloyd-Evans, Senior Lecturer, Division of Psychiatry, University College Psychiatry, University College London, UK. Email: b.lloyd-evans@ucl.ac.uk

doi:10.1192/bjp.2019.145 items from the CRT Fidelity Scale (a median of eight items per demonstrated that a service improvement programme, informed London, UK; Sonia Johnson, Professor of Social and Community Psychiatry, Division of

Aman Mehta, Senior Resident, Department of Psychiatry, Institute of Human Behavior and Allied Sciences, New Delhi, India. Email: aman18apri|@gmail.com

doi:10.1192/bjp.2019.146

\section{Authors' reply}

The logistical challenges of meeting India's mental healthcare needs are substantial, but not insurmountable. Many Indian clinicians are highlighting potential paths forward; often utilising and building upon pre-existing resources. Trained lay counsellors, ${ }^{1}$ and peer support workers ${ }^{2}$ are two good examples of what is possible. Financial and infrastructural investment is also essential particularly to facilitate treatment within the community; half-way homes, sheltered accommodation and supported accommodation are an unmet need.

The incorporation of Ayurveda, yoga and naturopathy, Unani, siddha and homoeopathy into the Mental Healthcare Act presents a unique opportunity. The reality on the ground is that many individuals with mental illness attend practitioners of traditional medicine, who are often highly skilled. ${ }^{3}$ The exclusion of traditional practitioners from the Act would have been unlikely to stop the use of such services; consequently, their inclusion facilitates their regulation and registration. It brings their establishments under the remit of the Mental Healthcare Act and provides individuals attending their services with the same patient-centred rightsbased protections

Section 106 of the Mental Healthcare Act prohibits mental health professional (including traditional practitioners) from recommending 'any medicine or treatment not authorised by the field of his profession'. This will hopefully prevent all healthcare providers from practising outside of their field of expertise. In meeting the high standards put forward in the Mental Healthcare Act traditional practitioners may need to increasingly collaborate with psychiatry and this presents all parties with opportunities to enhance their treatments and better serve their patients.

1 Patel V, Weiss H, Chowdhary N, Naik S, Pednekar S, Chatterjee S, et al. Lay health worker led intervention for depressive and anxiety disorders in India: impact on clinical and disability outcomes over 12 months. Br J Psychiatry 2011; 199: 459-66.

2 Pathare S, Kalha J, Krishnamoorthy S. Peer support for mental illness in India: an underutilised resource. Epidemiol Psychiatr Sci 2018; 27: 415-9.

3 Thirthalli J, Zhou L, Kumar K, Gao J, Vaid H, Liu H, et al. Traditional, complementary, and alternative medicine approaches to mental health care and psychological wellbeing in India and China. Lancet Psychiatry 2016; 3 : 660-72.

healthcare to all sections of society. The government also intends to 'integrate mental health services into general healthcare'. As India has a large population of 1.3 billion people there might be certain difficulties in implementing the Act.

As we all are aware, there is a dearth of psychiatrists and mental health staff to cater for the needs of the large population. We also know that there are remedies and treatments available in Ayurveda and other traditional methods that are practised in India. I would like to ask the authors' view about how they would recommend the Indian government and the Indian Psychiatric Society addresses the needs of people with mental illness when there is a big treatment gap across the country. It will also be challenging to incorporate the Mental Health Care Act for remedies and management options provided by Ayurveda, yoga and naturopathy, Unani, siddha and homeopathy establishments in the coming days. What would be the authors' view about how India, with a diverse culture, can align its mental health services so that they are at par with higher-income economic countries.

1 Duffy RM, Kelly BD. The right to mental healthcare: India moves forward. $\mathrm{Br} J$ Psychiatry 2019; 214:59-60.
Richard M. Duffy, Consultant Psychiatrist, PhD Researcher, Trinity College Dublin, Ireland. Brendan D. Kelly, Professor of Psychiatry, Trinity College Dublin, Ireland. Email: duffyrm@gmail.com

doi:10.1192/bjp.2019.147

\section{Scapegoating mentally ill people}

Thank you for publishing the interesting debate on the ethics of diagnosing psychiatric disorders in public figures. ${ }^{1}$ Langford correctly draws attention to the inevitable stigmatisation of all those with mental illness which such public diagnoses would entail, but arguably a more pertinent issue here is that of scapegoating.

French intellectual Rene Girard (1923-2015) claimed that scapegoating, although eschewed by modern ethics, was an important adaptation in human evolution, inducing the unanimity of 'all against one', and thus strengthening group cohesion and curtailing internecine violence. ${ }^{2}$ Applying this Girardian anthropology, I have recently proposed the archetypal scapegoat hypothesis ${ }^{3}$ on the 